\title{
H-Mordenite as a Molecular Reactor for the Isopropylation of Biphenyl
}

\author{
Stalin Joseph, Yoshihiro Sugi, Kavitha Ramadass, Baskaran Rajesh, and Ajayan Vinu
}

\begin{abstract}
The dealuminated H-Mordenite (MOR) was used as a molecular reactor for the selective formation of the least bulky 4,4'-diisopropylbiphenyl (4,4'-DIPB) in the isopropylation of biphenyl (BP). The selective formation of 4,4'-DIPB through 4-isopropylbiphenyl (4-IPBP) was observed due to the differentiation from the other bulky isomers by the MOR channels. 4,4'-DIPB selectively allowed the transition state of 4-IPBP by their steric restriction of the channels. The selectivity for 4,4'-DIPB decreased at high temperatures and/or when a large amount of the catalyst was used. These are due to the isomerization of 4,4'-DIPB, once formed in the channels, to a thermodynamically stable 3,4'- and 3,3'-DIPB at the external acid sites. Y-zeolite (FAU) and zeolite- $\beta$ (BEA) possess wide channels to accommodate the bulkier isomers, which allow the transition states of IPBPs to the DIPBs with 2- and 3-isopropyl moieties.
\end{abstract}

Index Terms - Biphenyl, isopropylation, molecular reactor, mordenite, shape-selective catalysis.

\section{INTRODUCTION}

Friedel-Crafts alkylation of aromatic hydrocarbons with Lewis acid catalysts, such as $\mathrm{AlCl}_{3}$ occurs under kinetic and/or thermodynamic controls: electron rich active positions of the reactant molecules are easily attacked in the former cases. The thermodynamic properties of the isomers lead to the more stable isomers [1]. Inorganic solid acids, such as zeolites and amorphous silica-alumina, also serve as the active catalysts for the alkylation [2]-[6]. Particularly, the catalysis of the zeolites is due to their confined microporous environments, and can lead to the third reaction control by the steric restriction by their pores and channels. The steric restriction by the channels of some zeolites invokes the selective formation of the least bulky isomers by shape-selective catalysis [3]-[6]. Thus, zeolites can work as intelligent materials for a molecular reactor if the steric restriction of the channels works effectively by their characteristic steric structures.

The isopropylation of biphenyl (BP) has been investigated by many researchers over various zeolites. Different isomers can be obtained by varying the types of zeolite [5-13]. We have observed the selective formation of 4,4'-DIPB, the least

bulky isomers, over H-mordenite (MOR). However, the other large pore zeolites, such as Y-zeolite (FAU) and zeolite- $\beta$ (BEA) gave the products with 2- and 3-isopropyl moieties under kinetic and/or thermodynamic controls

Manuscript received February 9, 2018; revised March 30, 2018.

The authors are with Future Industries Institute, University of South Australia, Mawson Lakes, 5095 SA, Australia (e-mail: stalinjoseph143@gmail.com, kavitha.ramadass@gmail.com, vinu.ajayan@gmail.com).
[5]-[11]. We have proposed that the difference is due to the microporous nature of these zeolites, and that only MOR can afford the reaction sites for selective formation of the least bulky 4,4'-DIPB in the channels [6]-[10]. On the other hand, the other zeolites can accommodate the bulky isomers in their pores and channels, resulting in the formation of the bulkier products with 2- and/or 3-isopropyl moieties [11].

In this paper, we discuss the natures of the shape-selective catalysis of MOR with 12-membered straight channels in the isopropylation of BP.

\section{EXPERIMENTAL}

\section{A. Catalysts and Reagents}

$\operatorname{MOR}\left(\mathrm{SiO}_{2} / \mathrm{Al}_{2} \mathrm{O}_{3}=10-220\right)$ and $\mathrm{FAU}\left(\mathrm{SiO}_{2} / \mathrm{Al}_{2} \mathrm{O}_{3}=30\right)$, where the value in parenthesis is $\mathrm{SiO}_{2} / \mathrm{Al}_{2} \mathrm{O}_{3}$ ratio of zeolites, were obtained from Tosoh Corporation, Tokyo, Japan, and calcined at $550{ }^{\circ} \mathrm{C}$ in an air stream before use. BEA was synthesized by following the procedures in our previous literature [14]. Some of the physicochemical properties of MOR have been discussed elsewhere [8,9]. All chemicals were commercially obtained and used without further purification.

\section{B. Isopropylation of BP}

The isopropylation of BP was carried out in a $100 \mathrm{ml}$ SUS-316 autoclave with magnetic agitation. The typical reaction conditions involved: BP (50 mmol), MOR ( $0.25 \mathrm{~g})$, propene pressure $(0.8 \mathrm{MPa})$, reaction temperature $\left(250^{\circ} \mathrm{C}\right)$, and reaction period $(4 \mathrm{~h})$. The autoclave containing BP and MOR was purged with nitrogen before heating. Once the reaction temperature was attained, propene was introduced into the autoclave with agitation, and the pressure was kept constant throughout the reaction. At the end of the reaction, the autoclave was quickly cooled to room temperature by immersing in a water bath, the catalyst was filtrated off, and the bulk products were diluted with toluene and acetone. The products were analyzed by a Shimadzu Gas Chromatograph, GC-14, equipped with an Ultra-1 capillary column $(0.25 \mathrm{~mm}$ x 30 - $60 \mathrm{~m}$; film thickness: $30 \mu \mathrm{m}$; Agilent Technologies, California, U.S.A.) by using FID detector.

The analysis of the encapsulated products in the catalyst used for the reaction was carried out as follows. The catalyst was washed well with toluene and acetone in order to remove the products on the external surface, and dried overnight at $60{ }^{\circ} \mathrm{C}$. About $50 \mathrm{mg}$ of the resultant catalyst was dissolved carefully using $3 \mathrm{~mL}$ of aqueous hydrofluoric acid (47 wt.\%) at room temperature. After the disappearance of solid residues, the solution was basified with solid potassium carbonate and the organic layer was extracted three times with $2 \mathrm{~mL}$ of dichloromethane. After the removal of the 
solvent in vacuo, the residue was dissolved in aliquot of acetone and subjected to GC analysis following the same procedure adopted for bulk products.

The yield of every product is calculated on the basis of the initial amount of $\mathrm{BP}$, and the selectivity for each isopropylbiphenyl (IPBP) and diisopropylbiphenyl (DIPB) isomers are expressed based on the total amounts of IPB and DIPB isomers, respectively.
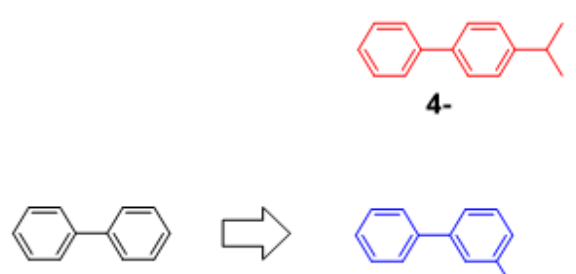

4-
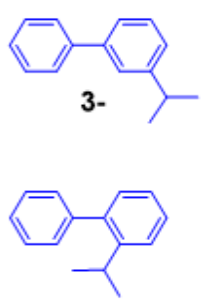

2

BP

\section{IPBP}

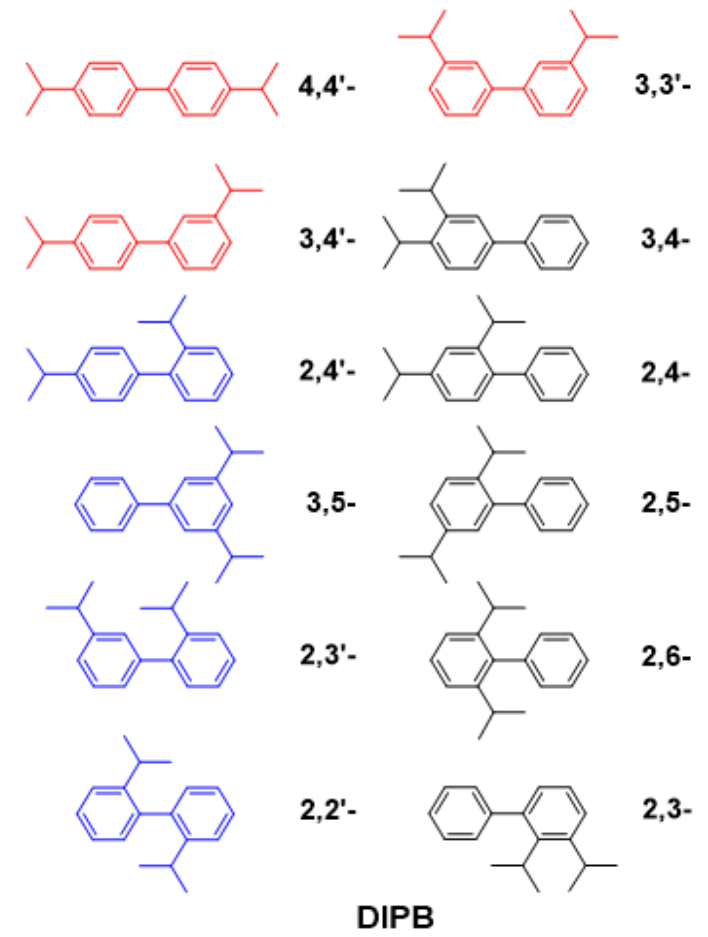

DIPB

Scheme 1. Possible IPBP and DIPB isomers in the isopropylation of BP over MOR. red: predominant isomers; blue: minor isomers; black: unidentified. Isomers.

\section{PRODUCTS IN THE ISOPROPYLATION OF BP}

Scheme 1 shows the possible IPBP and DIPB isomers that appear in the isopropylation of BP. The bulkiness of these isomers found as products is in the order: 4- $>3->2$ - for IPBP isomers and 4,4'- > 3,4'- > 3,3'> 2,x'- (x: 2-, 3-, and 4-) for DIPB isomers, because the isopropyl moieties influences their bulkiness. 2-IPBP and 2,x'-DIPB, the bulkiest isomers among the IPBP and DIPB isomers, respectively, are formed under the kinetic control by an electrophilic attack of the alkylating agent at the electron-rich 2-position of BP. On the other hand, 3-IPBP and 3,3'- and 3,4'-DIPB, thermodynamically more stable isomers, are preferentially formed under the thermodynamic control which works at higher reaction temperatures, during a long reaction, under low propene pressures, and/or with a large amount of catalyst. These controls work under sterically less hindered conditions. 4-IPBP and 4,4'-DIPB, the least bulky isomers, appears as the principal products by shape-selective catalysis only under the appropriate steric controls of the zeolite channels [5]-[10].

\section{Mechanism of Shape-Selective Catalysis in the ISOPROPYLATION OF BP}

Three types of mechanism of shape-selective catalysis were originally proposed by Sciscery [4] as shown in Scheme 2 for the isopropylation of BP. They depend on whether the pore size limits the entrance of the reactant molecules, the departure of the product molecules, or the formation of certain transition states.

A reactant selectivity mechanism (Mechanism I) occurs only when some of the molecules in the reaction mixture can enter and react in the pores. Only the least bulky 4-isopropylbiphenyl (4-IPBP) can enter the pores, resulting in the shape-selective formation of 4,4'-DIPB. However, 3- and 2-IPBP, which are too large to enter the pores, cannot react.

A restricted transition state selectivity mechanism (Mechanism II) occurs when certain reactions are prevented because the corresponding transition state would require more space than available inside the pores. The transition state to form 4,4' DIPB can fit the channels, resulting in the preferential formation of 4,4'-DIPB. However, the transition state to other bulky isomers is excluded because of their steric restriction with the zeolite channels.

A product selectivity mechanism (Mechanism III) occurs when some of the products formed in the catalyst pores are too bulky to diffuse out, resulting in their further conversion to less bulky molecules (e.g., by equilibration or cracking). DIPB isomers establish an equilibrium in the channels, and the least bulky 4,4'-DIPB leaves the pores due to its rapid diffusion. The other bulky products slowly diffuse out from the zeolite, and some of them may eventually deactivate the catalytic sites by blocking the pores.

These mechanisms are keys to achieve the highly shape-selective catalysis in the alkylation of BP [4,5-10]. In particular, it is important that the sizes of pores and channels of the zeolite precisely fit the molecular dimensions of the reactants and products. We discuss, in this paper, the 
relationships between the type of zeolite and bulkiness of the reagent in the alkylation of BP to elucidate the influence of the zeolite channels on the steric interaction of the transition states.
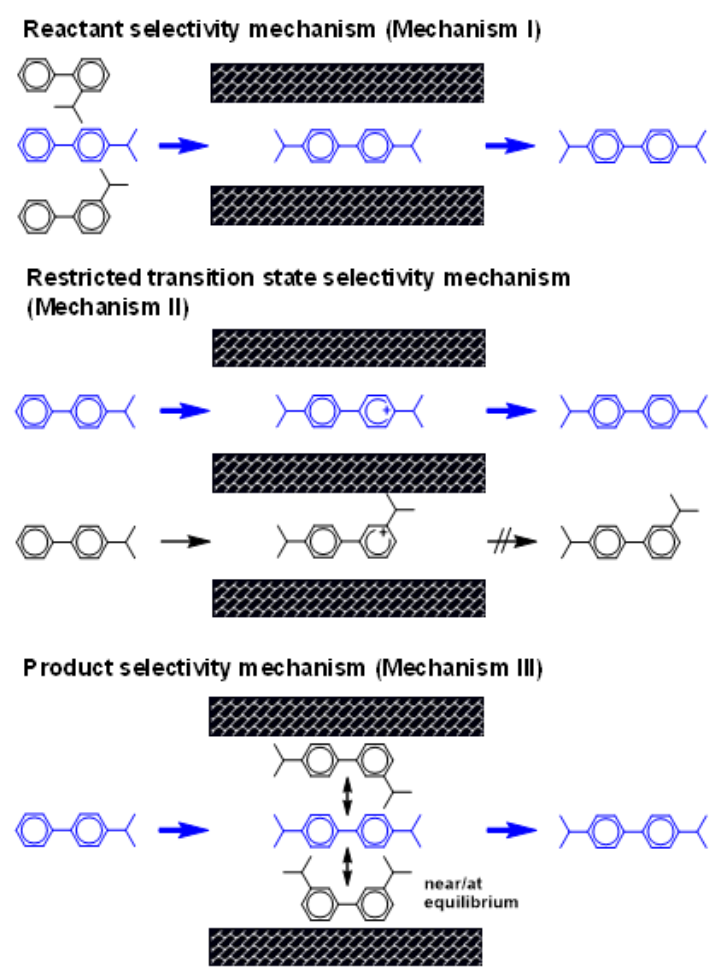

Scheme 2. Proposed mechanisms for the isopropylation of BP.

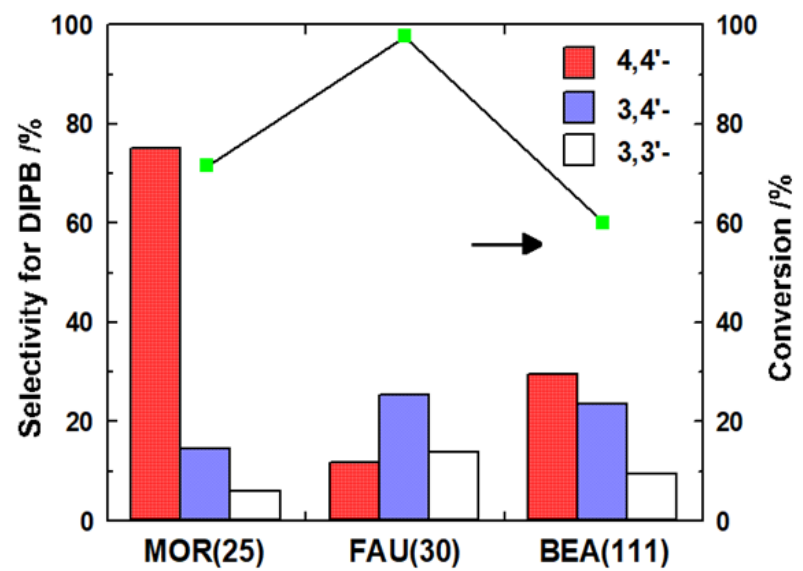

Fig. 1. The isopropylation of BP over large pore zeolites. Reaction conditions: catalysts, $0.5 \mathrm{~g}$; $\mathrm{BP}, 100 \mathrm{mmol}$; propene, $0.8 \mathrm{MPa}$; temperature, $250^{\circ} \mathrm{C}$; period, $4 \mathrm{~h}$.

\section{RESUlts AND DisCUSSION}

\section{A. Isopropylation of BP over Large Pore Zeolites}

Fig. 1 shows the catalytic activity and the selectivity of DIPB isomers in the isopropylation of BP over typical 12-memebered zeolites, MOR, FAU, and BEA. The selective formation of the least bulky 4,4'-diisopropylbiphenyl (4,4'-DIPB) was observed only over MOR. However, FAU and BEA, which have 3-dimensional systems of 12-membered ring pores, gave low selectivity for 4,4'-DIPB. MFI with 10 -membered ring pores had a very low catalytic activity with low selectivity for 4,4 '-DIPB. This is due to the fact that the channels are too small for BP to enter, and the isopropylation can occur only at the external acid sites. The results suggested that the type of zeolites is the key for the highly selective formation of 4,4'-DIPB. We have focused on MOR for the elucidation of shape-selective catalysis in this paper.

\section{B. Isopropylation of BP over MOR}

\section{1) Dealumination}

Fig. 2 shows the effects of $\mathrm{SiO}_{2} / \mathrm{Al}_{2} \mathrm{O}_{3}$ ratio of $\mathrm{MOR}$ on the isopropylation of $\mathrm{BP}$. MORs with $\mathrm{SiO}_{2} / \mathrm{Al}_{2} \mathrm{O}_{3}$ ratios, of less than 20 , gave only $60 \%-70 \%$ selectivity for 4,4'-DIPB, accompanying the formation of bulky 2, x'-DIPB (2,2'-, 2,3'and 2,,4'-) isomers, and they were rapidly deactivated by coke deposition from the early stage of the reaction. MORs with $\mathrm{SiO}_{2} / \mathrm{Al}_{2} \mathrm{O}_{3}$ ratio of 220 had the highest catalytic performance among all MOR catalysts as shown in Figure 2.

The dealumination by acids, such as hydrochloric acid, is an important technique for the enhancement in the catalytic performance of MOR [15-18]. The catalytic activity for the isopropylation of BP was enhanced by retaining the nature of MOR by the dealumination, and the selectivity for 4,4'-DIPB was much improved due to the decreased formation of 2,x'-DIPB. The improvement in the catalytic performance is due to the reduced coke formation by the removal of excess acid sites upon dealumination. The accessibility to the channels is also much improved by the formation of mesopores even though the number of acid sites was decreased.

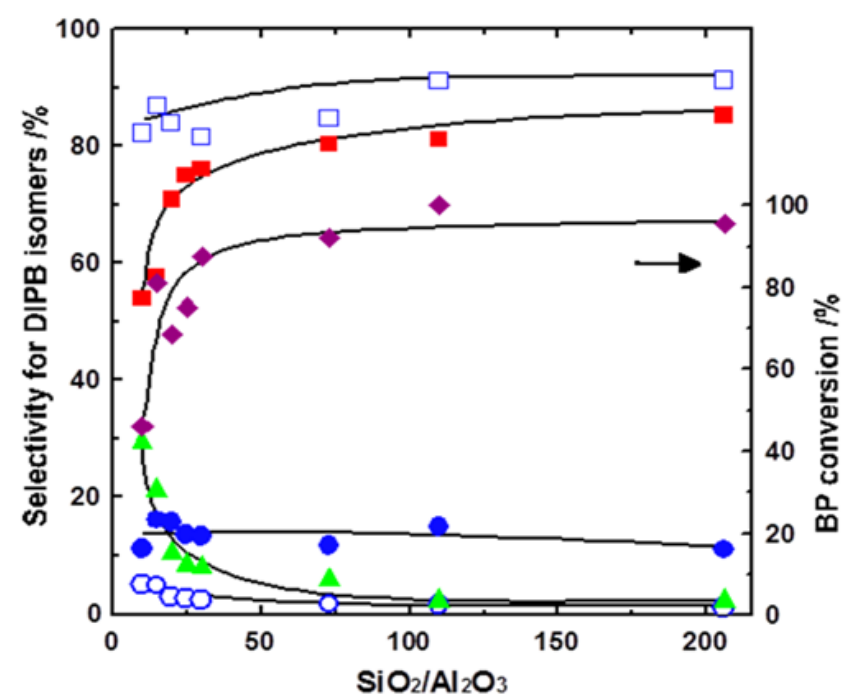

Fig. 2. Effects of the dealumination of MOR on the isopropylation of BP. Reaction conditions: BP: $200 \mathrm{mmol}$; $\mathrm{MOR}\left(\mathrm{SiO}_{2} / \mathrm{Al}_{2} \mathrm{O}_{3}=10-220\right)$ : $1 \mathrm{~g}$; propene pressure: $0.8 \mathrm{MPa}$; temperature, $250{ }^{\circ} \mathrm{C}$; period, $4 \mathrm{~h}$. Legend: bulk

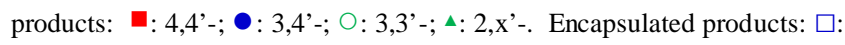

$$
4,4^{\prime}-;>\text { : BP conversion. }
$$

The selectivity for 4,4'-DIPB in encapsulated products was almost constant for all MORs, which means that the formation of 4,4'-DIPB occurred inside MOR channels even with the low $\mathrm{SiO}_{2} / \mathrm{Al}_{2} \mathrm{O}_{3}$ ratios. Low selectivity for the bulk products over MORs with the low $\mathrm{SiO}_{2} / \mathrm{Al}_{2} \mathrm{O}_{3}$ ratio was most likely through the non-selective formation of the bulky DIPB isomers at the external acid sites due to the rapid choking of the pore mouth by the coke formation. These results indicate that the dealuminated MOR channel can prevent the coke formation and catalyze the selective formation of 4,4'-DIPB in their steric circumstances. The dealuminated MORs were 
used for further investigation.

\section{2) Typical reaction profile}

Fig. 3 shows the reaction profile of the isopropylation of BP over MOR $\left(\mathrm{SiO}_{2} / \mathrm{Al}_{2} \mathrm{O}_{3}=220\right)$ at $250{ }^{\circ} \mathrm{C}$ with propene pressure of $0.8 \mathrm{MPa}$. 4,4'-DIPB was the predominant isomer among the DIPB isomers and its selectivity remained almost constant throughout the reaction. The formation of 4,4'-DIPB occurs by a consecutive mechanism: $\mathrm{BP}$ to 4-IPBP and 4-IPBP to 4,4'-DIPB. 4-IPBP, the predominant isomer in the first step, was maximized in the yield at $50 \%-60 \%$ of BP conversion; however, 3-IPBP increased spontaneously even in the late stages. Thus, there is a difference in the reactivity among the IPBP isomers: only 4-IPBP works as a precursor of 4,4'-DIPB, and 3-IPBP is excluded from the precursor of DIPB isomers. The selectivity for 4,4'-DIPB from 4-IPBP was higher than those for 4-IPBP from $\mathrm{BP}$ at the early stage (60\% for 4-IPBP and $85 \%$ for 4,4'-DIPB): this is due to the increased steric interaction of the isopropyl moiety of 4-IPBPwith the MOR channels. These results indicate that the MOR with channels can work for the highly shape-selective formation of 4,4'-DIPB in the isopropylation of BP.

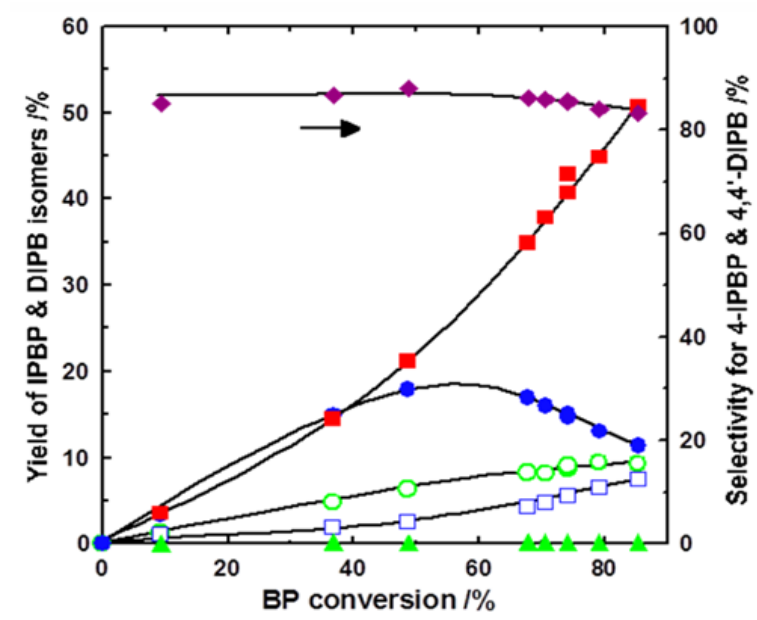

Fig. 3. The profile of the isopropylation of BP over MOR. Reaction conditions: $\mathrm{BP}, 400 \mathrm{mmol}$; $\mathrm{MOR}\left(\mathrm{SiO}_{2} / \mathrm{Al}_{2} \mathrm{O}_{3}=10\right.$ - 220), 2 g; propene pressure, $0.8 \mathrm{MPa}$; temperature, $250^{\circ} \mathrm{C}$. Legend: yield: $\square$ : 4,4'-; $\square$ : 3,4’-DIPB; • : 4-IPBP; ○: 3-IPBP; $\quad \Delta$ : 2-IPBP; selectivity: $\diamond: 4,4$ ’-.

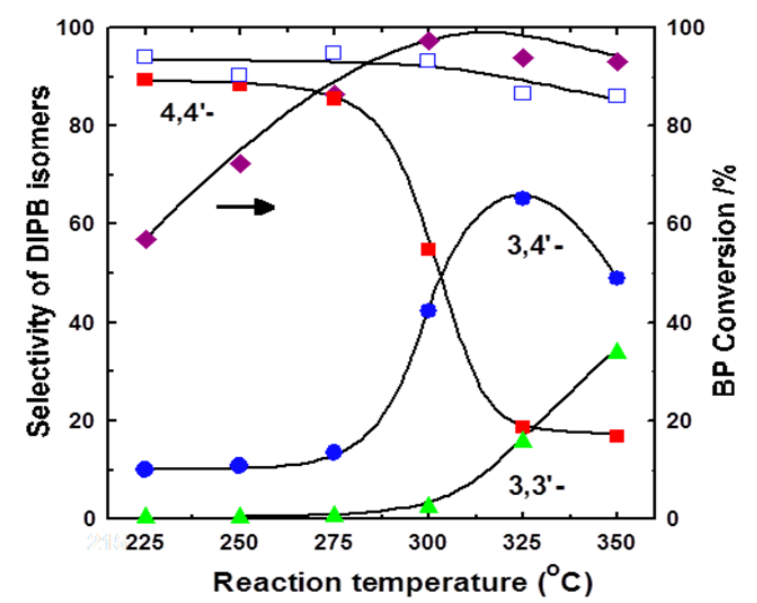

Fig. 4. Effects of reaction temperature on the selectivity for DIPB isomers and conversion of BP. Reaction conditions: BP: $200 \mathrm{mmol}$; MOR $\left(\mathrm{SiO}_{2} / \mathrm{Al}_{2} \mathrm{O}_{3}=206\right): 1 \mathrm{~g}$; propene: $0.8 \mathrm{MPa}$; period: $4 \mathrm{~h}$. Legend: bulk products: $\square$ : 4,4'-; $\bullet$ : 3,4'-; $\boldsymbol{\Delta}$ :3,3'-. Encapsulated products: $\square$ : 4,4'-. : BP conversion.

\section{3) Reaction temperature}

Fig. 4 shows the effects of reaction temperature on the selectivity for DIPB isomers over MOR(206). The selectivity for 4,4'-DIPB remained almost constant at low and moderate temperatures, i.e., below $250{ }^{\circ} \mathrm{C}$; however, they decreased at the temperatures higher than $275^{\circ} \mathrm{C}$ with an increase in the selectivity for 3,4'-DIPB. Further increase in the reaction temperature up to $350{ }^{\circ} \mathrm{C}$ enhanced the selectivity for 3,3'-DIPB with a decrease in the selectivity for 3,4'-DIPB. The decrease in the selectivity for $4,4^{\prime}$-DIPB is due to the isomerization of $4,4^{\prime}$-DIPB to the thermodynamically more stable 3,4'- and 3,3'-DIPB: first, 4,4'-DIPB to 3,4'-DIPB, then $3,4^{\prime}$-DIPB to $3,3^{\prime}$-DIPB.

The selectivity for 4,4'-DIPB in the encapsulated products remained constant even at high temperatures of $350{ }^{\circ} \mathrm{C}$. The discrepancy in the selectivity of bulk and encapsulated products suggests the different roles of acid sites in internal and external surfaces. The external acid sites favor the isomerization of 4,4'-DIPB, once formed in MOR channels. However, the internal acid sites work for the formation of 4,4'-DIPB even at high temperatures, such as $350^{\circ} \mathrm{C}$.

\section{4) Roles of external acid sites}

Fig. 5 shows the effects of BP/MOR ratio (reciprocal of catalyst amount) on the selectivity for DIPB isomers in the isopropylation of BP over MOR(128). The selectivity for DIPB isomers was influenced by $\mathrm{BP} / \mathrm{MOR}$ ratios and reaction temperatures. The selectivity for 4,4'-DIPB was remained constant as high as $80 \%-90 \%$ at $200{ }^{\circ} \mathrm{C}$ in the range of BP/MOR ratio: 20 - 3000, whereas the selectivity for 4,4'-DIPB decreased with a decrease in the ratio at 250 and $300^{\circ} \mathrm{C}$. The decrease in the selectivity for $4,4^{\prime}$-DIPB started at lower $\mathrm{BP} / \mathrm{MOR}$ ratios with an increase in the reaction temperature: 67 at $300{ }^{\circ} \mathrm{C}$ and 200 at $250{ }^{\circ} \mathrm{C}$. Further, the selectivity for 3,3'-DIPB increased with a decrease in those for $3,4^{\prime}$-DIPB at the ratios: $20-200$ at $300{ }^{\circ} \mathrm{C}$. The decrease in the selectivity for $4,4^{\prime}$-DIPB is ascribed to the isomerization of $4,4^{\prime}$-DIPB directing towards the thermodynamically more stable 3,4'- and 3,3'-DIPB.

The selectivity for 4,4'-DIPB in the encapsulated products were as high as $85 \%$ - $90 \%$ for all the ratios at 200 and $250{ }^{\circ} \mathrm{C}$, and also remained high: $75 \%$ at the ratios: 20 and 50 even at $300{ }^{\circ} \mathrm{C}$. These results mean that the formation of 4,4'-DIPB occurred inside the MOR channels even at the low $\mathrm{BP} / \mathrm{MOR}$ ratios, and that the isomerization of $4,4^{\prime}$-DIPB occurred at the external acid sites.

\section{Mechanistic Aspects of the Isopropylation of BP}

The results of the isopropylation of BP over MOR support that the catalysis occurs in the elliptical straight channels of $12-\mathrm{MR}$ pore entrances $(0.67 \times 0.71 \mathrm{~nm})$. The diagonal side pockets of 8 -MR pore entrances $(0.29 \times 0.57 \mathrm{~nm})$ do not concern in the catalysis since BP cannot diffuse through them. The catalysis proceeds by two consecutive steps: BP to IPBP isomers and 4-IPBP to 4,4'-DIPB by two mechanisms [5,6,8-10]. Mechanism I: the least bulky products, 4-IPBP among the IPBP isomers, and 4,4'-DIPB among the DIPB isomers, are predominantly formed inside the channels in two steps. Mechanism II: the exclusion of bulky isomers from the formation of the least bulky isomers by the steric interaction with the channels. BP yields predominantly 4-IPBP due to 
the steric restriction of transition states by the Mechanism I in the first step. Among the IPBP isomers, 4-IPBP works as only a precursor of 4,4'-DIPB, and bulky 2- and 3-IPBP were excluded by MOR channels through Mechanism II in the second step. The highly selective formation of 4,4'-DIPB occurs by synergism of Mechanisms I and II, particularly, due to an increase in the steric interaction of 4-isopropyl moiety of 4-IPBP and the exclusion of bulky 2- and 3-IPBP in the second step.

The encapsulated products are the "finger prints" of the catalysis inside the zeolite. These results indicate that the internal and external acid sites have different roles on the catalysis due to their different steric circumstances. The internal acid sites work for shape-selective formation of 4,4'-DIPB through Mechanisms I and II under all conditions. The external acid sites are not active under moderate reaction conditions; however, they are active for the non-selective catalysis under severe reaction conditions. Thus, the isomerization of 4,4'-DIPB, once formed inside the channels, occurred at the external acid sites. Further, we must note that Mechanism III, through which the least bulky 4,4'-DIPB forms predominantly by its rapid diffusion among the mixtures of DIPB isomers, does not work significantly in the selective formation of 4,4'-DIPB because 4,4'-DIPB was highly selective among encapsulated products.
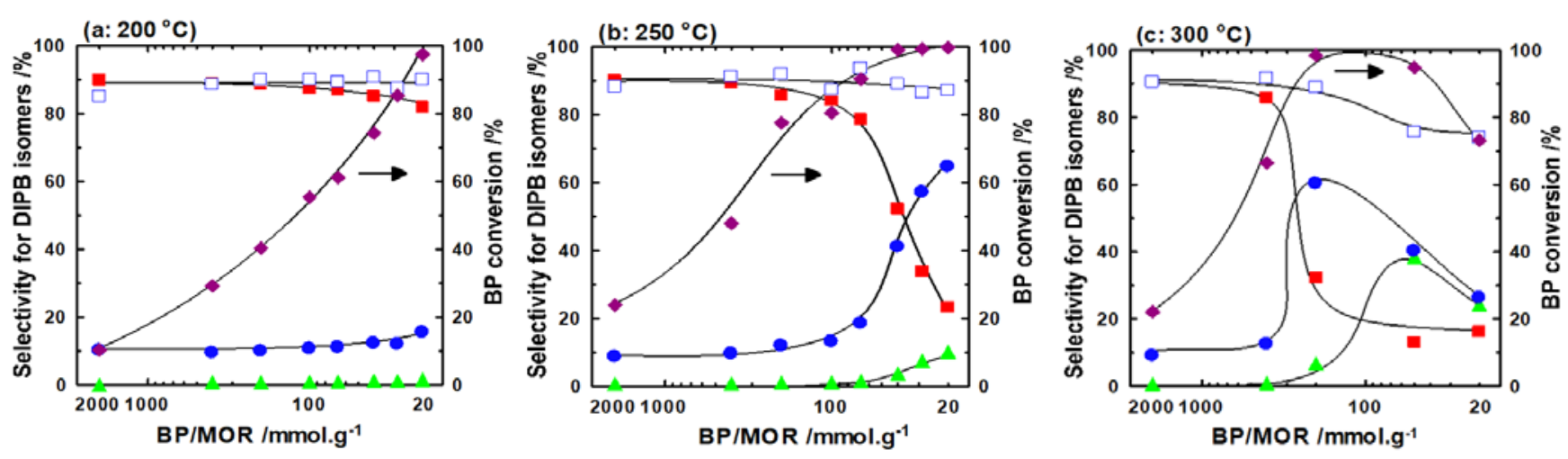

Fig. 5. Effects of BP/MOR ratio on the isopropylation of BP. Reaction conditions: $\mathrm{BP}, 200 \mathrm{mmol} ; \mathrm{BP} / \mathrm{MOR}\left(\mathrm{SiO}_{2} / \mathrm{Al}_{2} \mathrm{O}_{3}=128\right)$ : $20-2000 \mathrm{mmol} / \mathrm{g}$; temperature: (a) $200{ }^{\circ} \mathrm{C}$, (b) $250{ }^{\circ} \mathrm{C}$, and (c) $300{ }^{\circ} \mathrm{C}$; propene: $0.8 \mathrm{MPa}$; period: 4 h. Legends: see Fig. 4

Propene adsorption plays an important role in the catalysis at both of internal and external acid sites. On one hand, the strongly adsorbed propene on external acid sites hinders the access of BP, IPBP, and DIPB isomers to the acid sites under moderate reaction conditions, and prevents non-selective reactions, thereby resulting in the selective formation of 4,4'-DIPB at the internal acid sites. On the other hand, the vacant acid sites appear under severe reaction conditions such as high reaction temperature and/or by the use of large amount of the catalyst. Propene and 4,4'-DIPB can adsorb competitively on such vacant external acid sites. 4,4'-DIPB, directly adsorbed on such external acid sites, was isomerized to 3,4'- and 3,3'-DIPB. However, propene adsorbed on the internal acid sites cannot hinder the access of BP and IPBP isomers to the acid sites due to the steric limitations, thereby resulting in the shape-selective formation of 4-IPBP and 4,4'-DIPB.

The results from the current studies show that the shape-selective formation of 4,4'-DIPB occurred in MOR in the isopropylation of BP. However, the other 12-memebered zeolites, FAU and BEA, show no shape-selective natures with low selectivity for 4,4 '-DIPB. The difference is due to the structures of their channels: the latter two zeolites possess the channels that can accommodate the bulkier isomers and allow their formation through the transition states.

\section{CONCLUSIONS}

Dealuminated mordenite acts as a high performance molecular reactor with the shape-selective nature in the isopropylation of BP. Among the large pore zeolites, MOR, FAU, and BEA, the formation of $4,4^{\prime}$-DIPB was observed only over MOR. The encapsulated products "finger prints of the catalysis" showed high selectivity for 4,4'-DIPB under our conditions. These results indicate the selective formation of 4,4'-DIPB due to the shape-selective natures of MOR channels: the restriction of the transition state by steric interaction with the channels and the exclusion of bulky isomers from the channels. The decrease in the selectivity for 4,4'-DIPB under severe reaction conditions such as high reaction temperature and/or by the use of large amount of the catalyst is due to the isomerization of $4,4^{\prime}$-DIPB, once formed in the channels, at the external acid sites. These results clearly indicate that the MOR channels work as a shape-selective reactor.

Large pore zeolites, FAU and BEA, have enough space to accommodate the bulky DIPB isomers with 2- and 3-isopropy moieties, and allow the transition states to form these isomers, thereby, resulting in low selectivity for 4,4'-DIPB.

In conclusion, MOR works as a molecular reactor with the shape-selective natures. It is possible to design the catalysis of zeolites as a molecular reactor if the combination of reactants and products is tuned to fit the channels.

\section{REFERENCES}

[1] G. A. Olah, Friedel Crafts Chemistry, John Wiley, New York, N.Y. 1973.

[2] N. Y. Chen, W. E. Garwood, and F. G. Dwyer, Shape-selective Catalysis in Industrial Applications, 2nd ed., Marcel Dekker, New York, N. Y. 1996.

[3] P. B. Venuto, "Organic catalysis over zeolites: A perspective on reaction paths with micropores,” Micropor. Mater., vol. 2, pp. 297-411, 1994. 
[4] S. M. Csicsery, "Shape-selective catalysis in zeolites," Zeolites, vol. 4, pp. 202-213, 1984.

[5] Y. Sugi and Y. Kubota, "Zeolite-catalysed alkylation of polynuclear aromatics,” in: Catalysis, a Specialist Periodical Report, J. J. Spivey, ed., Royal Soc. Chem., vol. 13, pp. 55-84, 1997.

[6] Y. Sugi and A. Vinu, "Alkylation of biphenyl over zeolites: shape-selective catalysis in zeolite channels," Catal. Surv. Asia, vol. 19, pp. 188-200, 2015.

[7] T. Matsuzaki, Y. Sugi, T. Hanaoka, K. Takeuchi, H. Arakawa, T. Tokoro, and G. Takeuchi, "Shape-selectivity of zeolite catalysts in the alkylation of biphenyl," Chem. Express, vol. 4, pp. 413-416, 1989.

[8] Y. Sugi and T. Hanaoka, "Shape-selective alkylation of polynuclear aromatic hydrocarbons over H-mordenites," J. Jpn. Petro. Inst., vol. 41, pp. 193-206, 1994.

[9] Y. Sugi, S. Tawada, T. Sugimura, Y. Kubota, T. Hanaoka, T. Matsuzaki, K. Nakajima, and K. Kunimori, "Shape-selective isopropylation of biphenyl over H-mordenites. Relationships of bulk products and encapsulated products,” Appl. Catal. A: Gen., vol. 189, pp. 251-261, 1999.

[10] C. Anand, T. Sugimura, K. Komura, Y. Kubota, J. -H. Kim, G. Seo, A. Vinu, and Y. Sugi, "The isopropylation of biphenyl over H-mordenite Roles of 3- and 4-isopropylbiphenyls," Korean J. Chem. Eng., vol. 30, pp. 1043-1050, 2013.

[11] G. Takeuchi, H. Okazaki, T. Kito, Y. Sugi, and T. Matsuzaki, "Isomer selectivity in isopropylation of biphenyl over solid acid catalysts," $J$. Jpn. Petro. Inst., vol. 34, pp. 242-247, 1991.

[12] G. S. Lee, J. J. Maj, S. C. Rocke, and J. M. Garcés, "Shape selective alkylation of polynuclear aromatics with mordenite-type catalysts: A high yield synthesis of 4, 4'-diisopropylbiphenyl," Catal. Lett., vol. 2, pp. 243-247, 1989.

[13] T. Matsuda and E. Kikuchi, "Selective synthesis of 4,4'-diisopropylbiphenyl using mordenite catalysts," Stud. Surf. Sci. Catal., vol. 83, pp. 295-302, 1994.

[14] Pusparatu, Y. Haga, K. Aoyama, K. Komura, Y. Kubota, Y. Nishimura, and Y. Sugi, "Isomerization and cracking of hexane over beta zeolites synthesized by dry gel conversion method,” J. Jpn. Petrol. Inst., vol. 55, 120-131, 2012.

[15] H. G. Karge and J. Weitkamp, "Untersuchungen an dealuminierten mordenit-katalysatoren,” Chem. Ind. Tech., vol. 58, pp. 946-959, 1986.

[16] S. Bhatia, J. Beltramini, and D. D. Do, "Deactivation of zeolite catalysts,” Catal. Rev. Sci. Eng., vol. 31, pp. 431-480, 1989-1990.

[17] M. Guisnet and P. Magnoux, "Coking and deactivation of zeolites: Influence of the pore structure," Appl. Catal., vol. 54, pp. 1-27, 1989.

[18] M. Sawa, M. Niwa, and Y. Murakami, "Acid-leached dealuminated mordenite: effect of acid concentration on catalyst life in methanol conversion,” Appl. Catal., vol. 53, pp. 169-181, 1989.

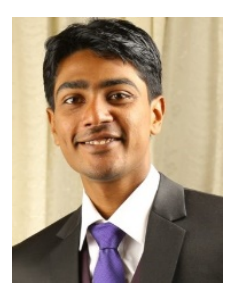

Stalin Joseph is originally from Trivandrum, Kerala, India. He did his bachelor's in Chemistry in Mar Ivanios College, Trivandrum. He completed his post-graduation from Vels University, Chennai. Currently he is on verge of completing his Ph.D in the field of functionalized nanoporous carbon electrodes for supercapacitors under the supervision of Prof. Ajayan Vinu in the University of South Australia.

During his masters' he joined AIBN, UQ summer internship program in 2012 and successfully completed the project on "Synthesis of novel nanoporous carbon nitride using MOF as template". During his Ph.D he was mainly focusing on synthesis of functionalized carbon electrodes from cheap carbon sources like coke, milk and juice for supercapacitor applications.

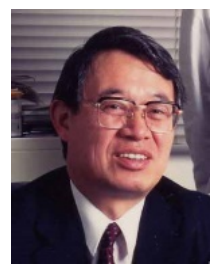

Yoshihiro Sugi is now a visiting professor in Future Industries Institute, University of South Australia, Mawson Lakes, 5095, SA, Australia on leave from
Faculty of Engineering, Gifu University, Gifu 501-1193, Japan. He got Ph.D in engineering at Tohoku University, Sendai, Japan in 1971. He joined in National Institute of Materials and Chemical Research (currently, Advanced National Institute of Science and Technology) in 1971. He moved to Faculty of Engineering, Gifu University, Gifu, Japan in 1995. He is now an emeritus professor of Gifu University. He stayed as a visiting professor in Advanced Institute of Bioengineering and Nanotechnology, The University of Queensland, Brisbane, QLD, Australia during 2011-2013. He has published more than 350 papers and got more than 100 patents until now. His research interests are catalysis in organic synthesis and in energy and environmental scenes.

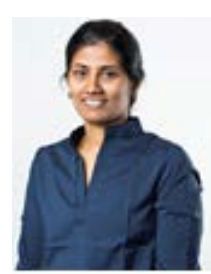

Kavitha Ramadass is a post-doctoral researcher in professor Ajayan Vinu's group at the Future Industries Institute, University of South Australia, Adelaide, Australia. She received Ph.D degree (2011) through International Postgraduate Research Scholarship at the Centre for Environmental Risk Assessment and Remediation (CERAR), University of South Australia. Her Ph.D research topic was on ecotoxicology and bioremediation of total petroleum hydrocarbons. After the completion of Ph.D, she remained in UniSA for her post-doctoral position, worked in multiple projects on bioremediation of hydrocarbon-contaminated soils funded by BHP Billiton Iron Ore (BHPBIO) and Co-operative Research Centre for Contamination Assessment and Remediation of the Environment (CRC CARE). Now she is focusing on developing on not only the innovative and efficient technologies on environmental remediation, through synthesis of multifunctional nanomaterials but also catalytic nanomaterials for the production of fine chemicals from $\mathrm{CO}_{2}$ molecules.

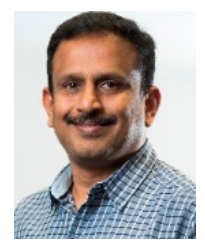

Baskaran Rajesh is a post-doctoral researcher in Prof. Ajayan Vinu's group at the Future Industries Institute, University of South Australia. He received Ph.D (2002) from Anna University, India on the synthesis, characterisation and catalytic application of microporous and mesoporous aluminophosphate based molecular sieves. He possess 12 years of experience in the e-publishing domain and had led a team of of copy editors, managed end-to-end operations of a typesetting set-up, and represented a quality assurance team for different major typesetting organizations serving globally acclaimed publishers like Elsevier, Nature Publishing Group, etc., in science, technology and medicine (STM). His current research focuses on the synthesis and characterization of novel mesoporous carbon nitrides prepared using mixed precursors for catalysis, photocatalysis and energy storage applications.

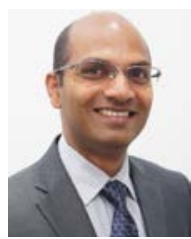

Ajayan Vinu is a professor of Nanomaterials at the Future Industries Institute, University of South Australia. He was working as a full professor and Australian Research Council Future Fellow at the University of Queensland. Prior coming to Australia, he had been working as a senior researcher and research group leader at the National Institute for Materials Science (NIMS), Tsukuba, Japan since 2006 after he successfully completed two years of the ICYS fellowship at the same institute and a few years of research at the Technical University of Kaiserslautern (TUK), Germany. During these 15 years of research, Prof. Vinu has made a tremendous contribution in the field of nanoporous materials and their application in sensing, energy storage, fuel cells, adsorption and separation, and catalysis. His contribution in the field of nanoporous materials is also clearly reflected by his international ranking by Science Watch as one of the top 15 researchers in the field and has led to more than 300 original research papers in high impact factor journals with ca. 12,500 citations and an H-index of 59 . 